\title{
Boosting exposure and response prevention with imagery-based techniques: a case study tackling sexual obsessions in an adolescent
}

\author{
A. Lau-Zhu ${ }^{1,2 *}$ (D) A. Farrington ${ }^{3}$ and C. Bissessar ${ }^{3}$ \\ ${ }^{1}$ Oxford Institute for Clinical Psychology Training and Research, Medical Sciences Division, University of Oxford, Oxford, \\ UK, ${ }^{2}$ Division of Psychiatry, Department of Brain Sciences, Imperial College London, London, UK and ${ }^{3}$ Anxiety \& Depression \\ Pathway, Children and Adolescent Mental Health Services, Berkshire Healthcare Foundation Trust, Bracknell, UK \\ ${ }^{\star}$ Corresponding author. Email: a.lau-zhu@imperial.ac.uk
}

(Received 22 April 2021; revised 17 November 2021; accepted 13 December 2021)

\begin{abstract}
Sexual obsessions are common in adolescents with obsessive compulsive disorder (OCD), but how to address these obsessions in a developmentally sensitive manner remains under-explored. This report presents the case of an adolescent who experienced unwanted sexual imagery, undergoing conventional exposure and response prevention, which was subsequently augmented with imagerybased techniques. This approach was associated with remission in symptoms of OCD and marked improvements in symptoms of anxiety and depression. The imagery-based approach was well received and valued as key to treatment success by the adolescent. This raises the tantalising possibility that working directly with images can fuel treatment innovation in tackling sexual (and non-sexual) obsessions in youth OCD.
\end{abstract}

\section{Key learning aims}

(1) Sexual obsessions are common in adolescent obsessive compulsive disorder (OCD).

(2) Little guidance is available on how to conduct exposure and response prevention sensitively for sexual obsessions in adolescent OCD.

(3) Imagery-based techniques can be used effectively for reducing sexual obsessions.

(4) Imagery-based techniques delivered by videoconferencing can be acceptable for young people.

Keywords: adolescents; exposure and response prevention; intrusive cognitions; mental imagery; OCD; sexual obsessions

\section{Introduction}

In this report, a 15-year-old adolescent completed a course of cognitive behavioural therapy (CBT) for obsessive compulsive disorder (OCD). Although he experienced obsessions and compulsions with a range of themes, his most troubling concern involved sexual obsessions - in the form of intrusive, visual mental imagery - depicting unwanted same-sex sexual acts. Following evidencebased guidelines (National Institute for Health and Care Excellence, 2005), exposure and response prevention (ERP) was initially employed and later augmented with imagery-based techniques, inspired by innovations in the CBT literature in adults (Hackmann et al., 2011; Holmes et al., 2019).

(C) The Author(s), 2022. Published by Cambridge University Press on behalf of the British Association for Behavioural and Cognitive Psychotherapies. This is an Open Access article, distributed under the terms of the Creative Commons Attribution licence (https:// creativecommons.org/licenses/by/4.0/), which permits unrestricted re-use, distribution, and reproduction in any medium, provided the original work is properly cited. 


\section{Literature review}

OCD is characterised by the experience of persistent and uncontrollable obsessional thoughts alongside compulsive acts, which are aimed at reducing the distress associated with obsessions (American Psychiatric Association, 2013). While individuals with OCD experience fears around key themes such as contamination, harm, need for symmetry and so forth, sexual obsessions (e.g. paedophilia, unwanted homosexual acts, incest, etc.) are also common (Gordon, 2002; Pinto et al., 2008; Rachman, 2007; Ruscio et al., 2010; Williams et al., 2014; Williams and Farris, 2011). Adolescence is a period marked by the development of sexual feelings and sexual identity, yet there is limited literature on sexual obsessions in youth OCD. Up to a quarter of young people (aged 8-17 years) with OCD are estimated to experience sexual obsessions, with an increased likelihood associated with being male and higher OCD severity (Fernández De La Cruz et al., 2013). Sexual obsessions can be associated with shame and stigma, leading to symptom concealment and thus potential mis-identification (Glazier et al., 2015). Relative to other symptom dimensions of OCD, the presence of sexual obsessions has been linked with poorer treatment outcomes (Williams et al., 2011b).

The gold-standard treatment for OCD across the lifespan is CBT (National Institute for Health and Care Excellence, 2005). CBT for youth OCD has also been shown to be effective beyond traditional face-to-face settings, rapidly expanding into other delivery formats, including via the internet (Lenhard et al., 2017). This development is crucial to meet the increasing demand for evidence-based psychological treatments to tackle youth mental health globally (Patel et al., 2007). When sexual obsessions are present, however, it remains contested whether CBT for OCD is as effective (Fernández De La Cruz et al., 2013; Mataix-Cols et al., 2002) and whether it requires longer treatments (Grant et al., 2006) and more specialist clinicians (Fernández De La Cruz et al., 2013),

CBT for youth OCD is based on clinical models developed with adult populations (Turner, 2006). Contrary to the adult literature, however, CBT protocols for youth OCD prioritise the 'behavioural' component (i.e. ERP) and place less emphasis on traditional 'cognitive' techniques (March, 1995). ERP is thought to be the 'active ingredient' for treating OCD, but its underlying mechanisms warrant further research (Turner, 2006). Specifically to sexual obsessions, there is very little clinical guidance on how to conduct ERP appropriately in young people. Some relevant clinical case descriptions involving adults have included exposure to sexual materials such as pornography (Williams et al., 2011a), which raises ethical issues in young people.

Traditional cognitive techniques are often perceived as too 'complex' for young people, who can struggle to verbalise their thoughts (Turner, 2006). In addition to verbal-based thinking, however, cognitions can also take the form of mental imagery, especially for emotionally charged experiences (Hackmann et al., 2011; Holmes et al., 2019). Sexual obsessions are often imagery-based (Rachman, 2007), and the centrality of images is acknowledged in the diagnostic criteria for OCD (American Psychiatric Association, 2013). Nevertheless, direct work with images in OCD (beyond conventional imaginal exposure) by manipulating imagery content and/or properties is still in its infancy and is primarily limited to adults thus far (Maloney et al., 2019; Veale et al., 2015). From a neuroscientific perspective, imagery is a hypothesised preferred mode of thinking in young people (Burnett Heyes et al., 2013). If so, treatment innovation for youth OCD can leverage clinical science on imagery in established areas such as trauma (Ehlers and Clark, 2000), alongside other rapidly evolving imagery-focused approaches specific to youth mental health (Pile et al., 2021; Schwarz et al., 2020). 


\section{Introduction to the case}

Tom is a 15-year-old White British young person from a middle-class family living in England. He was referred to a Child and Adolescent Mental Health Service by his GP, who described that Tom is spending 6-12 hours a day with obsessions causing significant impairments to his quality of life.

\section{Presenting problems}

Tom and his mum Ruth attended the assessment together. Initially, he was too anxious to be seen alone. Tom reported experiencing thoughts that were frequent, vivid and distressing. Content of the images included setting fire to his house, stabbing someone, seeing Ruth sick in hospital and so forth, which he found deeply upsetting. Because these thoughts were repeated in his mind, Tom tried to 'get rid of them' or 'say the opposite thing'. Tom also reported needing to complete a number of overt compulsions at home and school. For example, he would wash his hands throughout the day and had a particular washing routine. Tom found it hard to resist the compulsions even though he recognised the thoughts were 'silly'. Tom also described increased palpitations, which made him think 'am I having a heart attack?'. He had developed involuntary eye twitches when he experienced 'bad thoughts'. Ruth said that Tom can be quite snappy at home when he is stressed. Ruth also recalled that Tom used to display 'strange' behaviours when he was younger, such as having to say goodbye five times when she left him in the playground at school. No thoughts of suicide or self-harm were reported, which remained the case throughout treatment. Sexual obsessions were not reported at this stage.

\section{Method}

\section{Case conceptualisation}

A 5-P's framework (Macneil et al., 2012) was used to (i) help Tom and Ruth make sense of Tom's 'story' (shared verbally but depicted here visually; Fig. 1); (ii) inform suitability for a CBT-based approach; and (iii) aid the therapist in holding in mind the developmental context. A finer-grained cognitive behavioural formulation for OCD (Fig. 1), developed collaboratively later with Tom, informed the core therapeutic tasks targeting maintenance factors only. The exposition of the broader/longitudinal formulation is therefore necessarily brief, and the interested reader can refer to further details (Fig. 1).

Key family factors and life events throughout Tom's upbringing, particularly key bereavements (predisposing factors), might have enhanced Tom's perception that 'bad things will happen'. Tom's heart problems as a child (including frequent hospital visits and family stress) appeared to have contributed to his vulnerabilities to anxiety (DeMaso et al., 2017; Gonzalez et al., 2021), as exemplified by some of his earlier 'OCD-like' rituals. Additionally, the homophobic culture in the UK (for example, the use of 'gay' as an insult) may have reinforced the idea that sexual obsessions are 'unacceptable' when adolescents seek to 'fit in'.

The onset of Tom's difficulties coincided with him starting GCSEs (General Certificate of Secondary Education) and during a developmental stage with the increased importance of peers and sensitivity to peer rejection (precipitating factors). Tom experienced obsessions as uncontrollable and negative; he performed compulsions to relieve distress, but which were counterproductive (perpetuating factors; described in more detail later). As OCD expanded over Tom's life, it inevitably had a detrimental impact on his academic, social and home situation (presenting factors). Despite these difficulties, Tom has many individual and family strengths (protective factors), harnessed later in therapy. For example, he was highly motivated with ERP homework, which was further supported by his parents who would perform spontaneous ERP tasks alongside him at home (e.g. Ruth would lick the bottle of bleach and 
Pre-disposing

\begin{tabular}{|c|c|c|c|c|}
\hline $\begin{array}{c}\text { History of } \\
\text { depression and } \\
\text { anxiety in the wider } \\
\text { family (genetic and } \\
\text { environmental } \\
\text { risks) }\end{array}$ & $\begin{array}{c}\text { Age } 4- \\
\text { multiple } \\
\text { visits to the } \\
\text { doctor due to } \\
\text { a heart } \\
\text { problem }\end{array}$ & $\begin{array}{l}\text { Age } 7 \text { - bereavement of } \\
\text { Uncle (Mum's cousin) } \\
\text { who was very close and } \\
\text { died suddenly in his mid- } \\
\text { 30s from an undiagnosed } \\
\text { brain tumour }\end{array}$ & $\begin{array}{l}\text { Age } 12-\text { Dad's } \\
\text { struggles with } \\
\text { severe } \\
\text { depression after } \\
\text { losing his own } \\
\text { father }\end{array}$ & $\begin{array}{l}\text { Homophobic } \\
\text { culture in the } \\
\text { UK and use of } \\
\text { 'gay' as insult in } \\
\text { adolescent boys }\end{array}$ \\
\hline & & $\downarrow$ & & \\
\hline
\end{tabular}

\section{Protective}

\begin{tabular}{|c|}
\hline Supportive family; \\
popular in school; \\
sporty; academically \\
bright; hopes for the \\
future in sport \\
journalism; low risk \\
concerns; family holds \\
open-minded views \\
about homosexuality \\
\hline
\end{tabular}
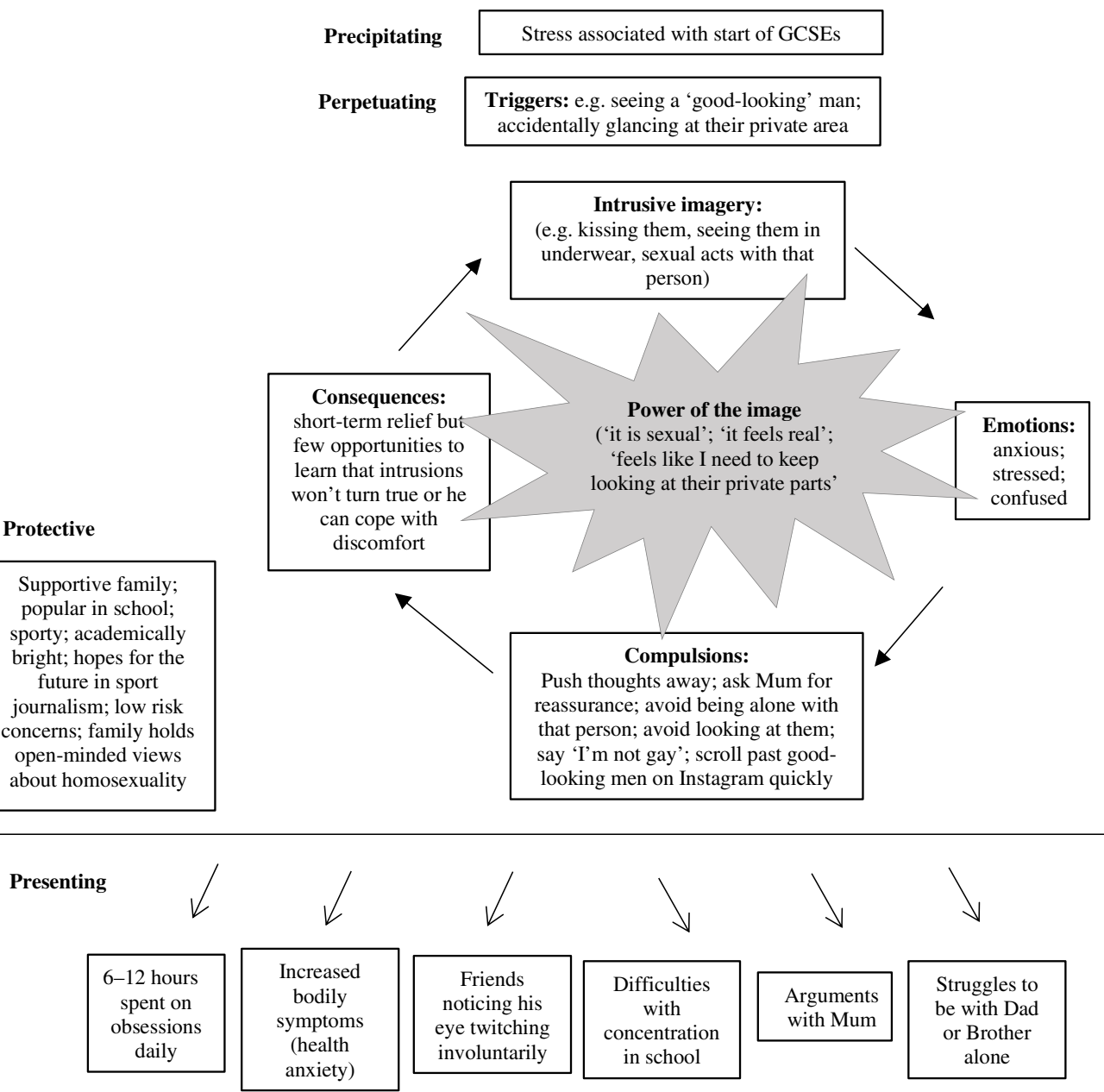

Figure 1. Developmental formulation for obsessive compulsive disorder with a focus on intrusive, unwanted, sexual mental imagery.

show Tom nothing deadly would happen; Tom would be suddenly asked by his dad if he could touch a table that has just been wiped with cleaning products).

OCD 'traps' (Derisley et al., 2008) were formulated for two identified core fears. For the fear of harming others, he explained that seeing a knife would trigger the image of 'stabbing Mum', leading to anxiety and stress; to relieve the distress, he would reassure himself 'he would never 
do this' and put the knife away. For the fear of contamination, wearing a jacket that has just been put on the floor would make him think'this is dirty', leading to anxiety; to relieve the distress, he would avoid zipping his jacket all the way up so there is no contact with his mouth and he would apply hand gel multiple times a day. In both 'traps', compulsions and avoidant behaviours led to a short-term reduction in anxiety, but his feared predictions were unchallenged, and his perceived inability to cope was reinforced, maintaining distressing intrusions.

In the first session on his own, Tom revealed for the first time that he also experienced sexual obsessions of same-sex acts - in fact, this core fear was the most troubling of all. Therefore, the 'trap' concerning sexual obsessions is depicted as central in the formulation (Fig. 1). Sexual obsessions were normally triggered by seeing other men, such as male friends, family members or 'good-looking' people in the media. His intrusive images involved sexual acts, such as kissing them. Tom found them confusing and stressful. Tom would avoid looking/ glancing at the 'crotch' area of other men, avoid being with them on his own, and selfreassure that he is straight, all of which only strengthened the sexual obsessions. As sexual exploration and identity forming is a normative task in adolescence, it seemed important to ascertain whether Tom found pleasure in experiencing such sexual images and whether there was a perceived judgement from self/others if he were gay.

As treatment progressed, the 'power of the image' (Holmes et al., 2019) was later incorporated to fully understand the persistence of the sexual obsessions, unlike the non-sexual counterparts (Fig. 1). This simple but targeted formulation provided the clinical rationale for boosting ERP with imagery-based techniques to dissolve the image's perceived power and fully tackle sexual obsessions.

\section{Goals}

Tom's goals were to (i) have fewer and less distressing sexual obsessions in the presence of other 'good-looking' men; (ii) spend less time completing compulsions (e.g. excessive handwashing); and (iii) to improve his sleep.

\section{Treatment delivery}

Tom attended 13 60-minute sessions of individual CBT. Two appointments were in person with the remaining sessions over videoconferencing due to safety measures imposed by the COVID-19 pandemic. Ruth attended the initial session only, as Tom expressed preference for sessions on his own, consistent with potential issues of shame surrounding sexual obsessions (Gordon, 2002). Ruth was involved in brief check-ins at mid- and post-treatment. Tom agreed to share his learning with Ruth at the end of each session to ensure that the family was 'on the same page'.

\section{Content of sessions}

In agreement with Tom, non-sexual obsessions were addressed first (sessions 1-7) to build his confidence prior to tackling sexual obsessions (sessions 8-12). Topics covered per session were as follows:

Sessions 1-2: Review of OCD symptoms; psychoeducation about anxiety; externalising OCD as a 'bully'; normalising intrusions; behavioural experiment to show the counterproductive effects of imagery suppression (Table 1); goal setting.

Sessions 3-7: Microformulation of OCD 'traps' for two non-sexual OCD themes (fear of harming others and fear of contamination); collaboratively developing the exposure hierarchy (and anxiety ratings $0-10$ per step); in vivo ERP (e.g. holding a knife against his palm; wiping his mouth after touching a bottle of bleach); daily ERP homework. 
Table 1. Imagery-based techniques used to target sexual (and other) obsessions

\begin{tabular}{|c|c|c|c|}
\hline $\begin{array}{l}\text { Imagery-based } \\
\text { techniques }\end{array}$ & $\begin{array}{l}\text { Obsessions } \\
\text { targeted }\end{array}$ & Application & Rationale \\
\hline $\begin{array}{l}\text { Image suppression } \\
\text { (and behavioural } \\
\text { experiment) }\end{array}$ & All obsessions & $\begin{array}{l}\text { In vivo demonstration of the } \\
\text { counterproductive effects of } \\
\text { suppression (Rachman, 2007) }\end{array}$ & $\begin{array}{l}\text { Justify the importance of } \\
\text { dropping this behaviour } \\
\text { during ERP }\end{array}$ \\
\hline Imaginal exposure & Sexual obsessions & $\begin{array}{l}\text { Identify a situation to trigger a } \\
\text { mental image and learn to } \\
\text { tolerate the resulting distress } \\
\text { while refraining from overt/ } \\
\text { covert compulsions (Gillihan } \\
\text { et al., 2012) }\end{array}$ & $\begin{array}{l}\text { Integration with ERP provides } \\
\text { a more developmentally } \\
\text { appropriate alternative } \\
\text { (e.g. vs in vivo exposure to } \\
\text { pornography) and is } \\
\text { recommended for fears } \\
\text { around changing in a } \\
\text { fundamental way about the } \\
\text { self (e.g. in relation to } \\
\text { sexuality) }\end{array}$ \\
\hline Imagery manipulation & Sexual obsessions & $\begin{array}{l}\text { Identify a situation to trigger a } \\
\text { mental image and introduce } \\
\text { elements to make it funny } \\
\text { (Holmes et al., 2019) }\end{array}$ & $\begin{array}{l}\text { Reinforce that 'an image is just } \\
\text { an image' }\end{array}$ \\
\hline Imagery interference & All obsessions & $\begin{array}{l}\text { Engage in visually engaging tasks } \\
\text { to compete with flooding of } \\
\text { unwanted images (Kavanagh } \\
\text { et al., 2005; Lau-Zhu et al., 2017) }\end{array}$ & $\begin{array}{l}\text { To efficiently manage images } \\
\text { temporarily and increase } \\
\text { self-efficacy }\end{array}$ \\
\hline $\begin{array}{l}\text { Imagery (cognitive) } \\
\text { diffusion }\end{array}$ & All obsessions & $\begin{array}{l}\text { Visualise each intrusion floating } \\
\text { gently as a leaf through a river } \\
\text { stream without trying to push } \\
\text { them away (Harris, 2009; Twohig } \\
\text { et al., 2010) }\end{array}$ & $\begin{array}{l}\text { Low-arousal strategy to reduce } \\
\text { imagery absorption and } \\
\text { promote stress reduction }\end{array}$ \\
\hline
\end{tabular}

Sessions 8-10: Microformulation on OCD 'trap' for sexual obsessions; psychoeducation (e.g. anxiety $v s$ arousal; normalising sexual obsessions); ERP incorporating imaginal exposure, which involved holding in mind and tolerating the unwanted sexual obsession imagery (Table 1); Tom identified triggering situations for the exposure hierarchy: (i) sharing details of the obsessions in therapy; (ii) looking at the face of a male model; (iii) looking at a shirtless model; (iv) staring at the model's underwear.

Although Tom's intrusions became less distressing, they remained frequent and continued to disrupt his schoolwork. Thus, he was introduced to the notion of imagery interference (Table 1). He identified easily accessible and visually engaging activities (e.g. playing a videogame; scrolling through Instagram on his phone) to cut his attention off the obsessive visual-based images and then return to his homework.

Sessions 11-12: Tom progressed much slower through the ERP hierarchy for sexual ( $v s$ nonsexual) obsessions. He was unable to dismiss the image because it was 'sexual' and seemed 'real'. Imaginal exposure was hence substituted with imagery manipulation (Table 1) to reduce the image's 'power'. Tom was introduced to the idea that he can modify what happens in the image (of 'seeing a male underwear model in bed') to whatever he wanted (akin to a 'film director') to make it less 'sexual'. He first followed the therapist's lead to imagine dressing up the model in a clown outfit. Tom then introduced his own 'funny' ideas. He brought two of his best friends in, and they all started jumping on trampolines, while the model rode a unicycle around the room with all of them shooting paint of multiple colours at each other. He later reported being able to apply the same 'film director' idea to sexual images involving other people.

Tom also reported being troubled by intrusions at nights, particularly when he was tired. As imagery interference strategies using his phone may be counterproductive for sleeping, 
an imagery defusion technique (see Table 1) was added to help him mindfully distance himself from his thoughts and help alleviate stress.

Session 13: Written relapse prevention plan; ideas for 'overlearning' were discussed, including ways to show he has 'conquered' the sexual obsessions (e.g. watching an age-appropriate gay-themed romantic film); working on endings.

\section{Design and outcomes}

\section{Primary measures}

The Children's Yale-Brown Obsessive-Compulsive Scale (CY-BOCS) was used to measure the severity of OCD at both the start and end of treatment. The CY-BOCS is a semi-structured interview for youth OCD that covers frequency, interference, distress, resistance and control of OCD symptoms (Scahill et al., 1997). It has good internal consistency, inter-rater reliability and convergent validity (Scahill et al., 1997). The CY-BOCS was administered by the treating clinician with both Tom and Ruth present each time.

Child and parent versions of the Revised Children's Anxiety and Depression Scale (RCADS) were used at initial assessment and start, mid and end of treatment. The RCADS (Chorpita et al., 2000) is a self-report questionnaire with six subscales to assess symptoms of depression and anxiety. It has good internal consistency (Chorpita et al., 2005), test-re-test reliability (Chorpita et al., 2000), convergent validity (Esbjørn et al., 2012) and concurrent validity (Chorpita et al., 2005).

\section{Secondary measures}

Goal-based outcomes were used to track progress towards individualised goals at both start and end of treatment, each on a scale from 0 (not achieved at all) to 10 (fully achieved; Law and Jacob, 2015). The Experience of Service Questionnaire (ESQ) was used to assess Tom's satisfaction with the service at the end of treatment (Brown et al., 2014). Idiosyncratic ratings were used to track belief related to the power of sexualised images ('It is sexual') from 0 (not true at all) to 100 (extremely true).

\section{Results}

CY-BOCS scores dropped from 22 (moderate) to 5 (below the clinical cut-off of 16) from start to end of treatment. This corresponds to a 77\% reduction in OCD symptoms, which is considered remission (Skarphedinsson et al., 2017).

According to the RCADS (Fig. 2), Tom's self-reported symptoms of anxiety and depression were above the clinical threshold ( $t$-score $>70)$ at assessment, start of and mid-treatment but had dropped below this threshold at end of treatment. Tom's symptoms, as reported by Ruth, were generally higher. Ruth, however, reported symptom reduction earlier than Tom - with scores already below the clinical threshold by mid-treatment.

The belief that his mental image 'was sexual' dropped from $90 \%$ to $0 \%$ after introducing the image manipulation technique in session 10 .

Tom's goals were rated as 1, 2 and 3 at the start of treatment, but 10, 9 and 8 at the end of treatment, reflecting high ratings of goal attainment. For the ESQ, he rated all items on satisfaction with care (e.g. 'If a friend needed similar help, I would recommend that they come to the service') as 'certainly true' and wrote in the free-text box 'for me everything was pretty perfect'.

\section{Discussion}

This report presented the case of a 15-year-old adolescent, Tom, who underwent 13 sessions of weekly CBT for OCD with unwanted sexual obsessions as his most distressing symptoms. 

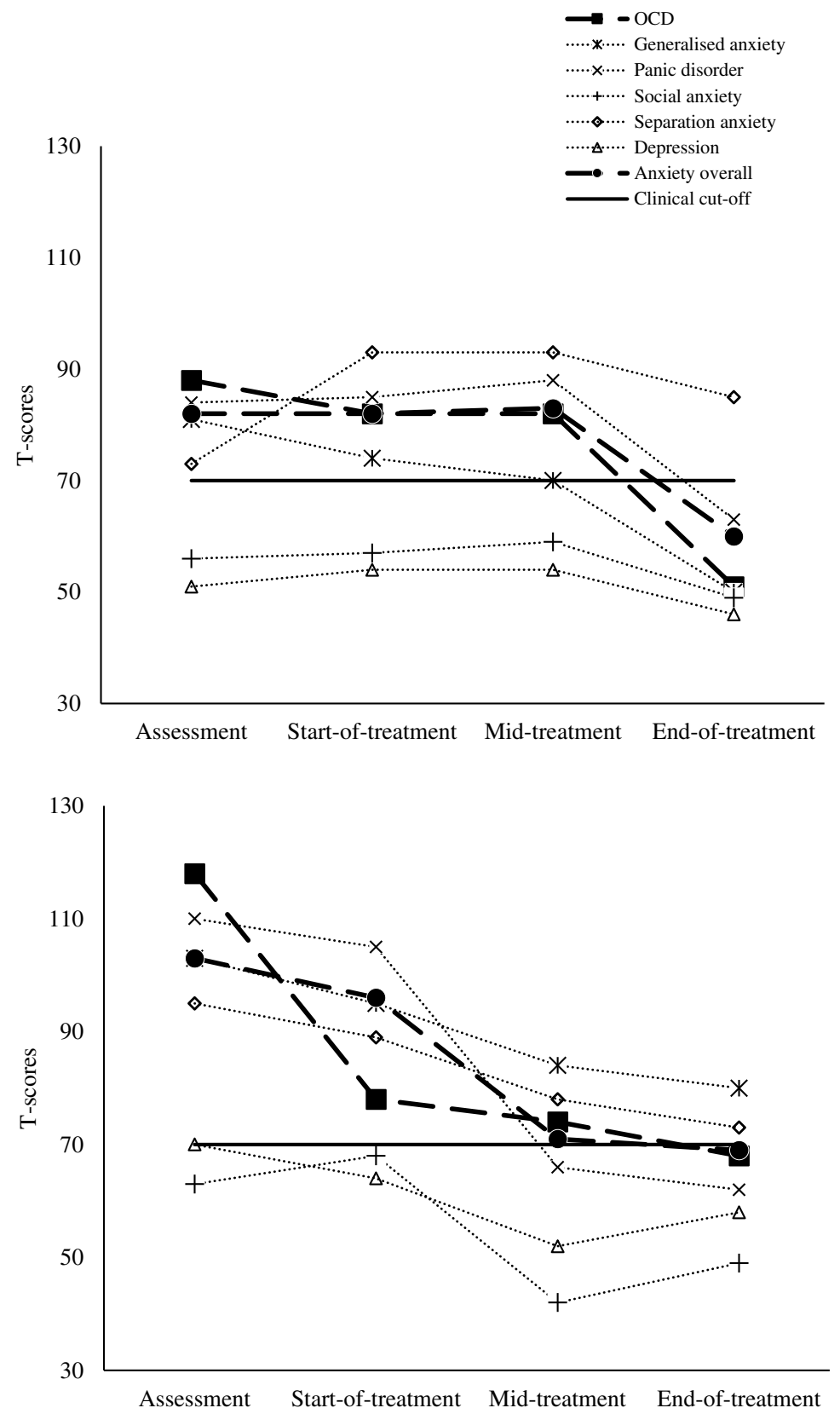

Figure 2. T-scores for the Revised Children Anxiety and Depression's Scale rated by the child (top panel) and the parent (bottom panel) from assessment through to treatment. OCD, obsessive compulsive disorder.

This was delivered by videoconferencing precipitated by a national lockdown in the UK due to the COVID-19 pandemic. The treatment protocol began with ERP - the conventional approach for youth OCD - and was later augmented with imagery-based techniques. Tom's scores on all primary outcome measures (CY-BOCS and RCADS) indicated symptom reduction throughout treatment, with scores indicative of remission towards the end. 
There are important issues to consider with our key outcome measures. While Tom's RCADS scores declined throughout treatment, his scores were overall higher when rated by Ruth. Such informant discrepancies are common (De Los Reyes et al., 2015). Higher parental ratings of adolescent anxiety have been associated with higher maternal anxiety (Fjermestad et al., 2017), which could partly reflect Ruth's heightened concerns about Tom's wellbeing (e.g. given his early experiences in the hospital) but possibly also her own ongoing worries related to the COVID-19 pandemic. Moreover, the CY-BOCS was administered by the treating clinician, which could have introduced a bias, although this is standard in routine care. Note, however, that this clinical interview was supplemented by the use of parent ratings and child self-reports producing converging results. Our multi-method assessment strengthened the observation that our intervention indeed successfully led to symptom reduction.

The 'power' of sexual imagery was understood as a key maintenance factor when ERP did not appear to produce treatment gains as rapidly as it had done for Tom's non-sexual obsessions. While conventional ERP does consider imagery somewhat (e.g. imaginal exposure), here, further imagery-based techniques were drawn from the CBT literature for adults (Hackmann et al., 2011; Holmes et al., 2019), targeting both the content and properties of the image. Image manipulation was used as an alternative to ERP, whereas imagery defusion and interference techniques provided Tom with tools for day-to-day symptom relief in relation to all obsessive images, which Tom reported as especially helpful for his sleep (goal 3). Overall, the imagery-based approach was associated with full goal attainment for reducing sexual obsessions (goal 1). Note that the image manipulation approach differed from recent descriptions of imagery rescripting in adults with OCD (Maloney et al., 2019; Veale et al., 2015), where aversive memories (from which intrusive images stemmed) were modified rather than the intrusive images per se. This memory-based approach was not employed here, as there was no indication that Tom's sexual obsessions were linked to early experiences.

What could have been the mechanisms underlying these imagery techniques? Of all the techniques used, image manipulation was key, as it was linked to in-session changes in belief ratings of the 'sexual' nature of the image. Changing the content of a specific image served as a springboard for Tom to understand the power of images and to modify his relationship to sexual images in general. Such change could be thought to occur at a meta-cognitive level (Fisher and Wells, 2008) because Tom was able to generalise this technique to other unwanted sexual images outside of sessions (e.g. other men), perhaps learning that 'an image is just an image'.

Mechanistic changes may have also occurred at the representational level, where new, nonthreatening images were formed to compete with the original sexual image, resulting in 'retrieval competition' (Brewin, 2006) as Tom encountered triggers. What might be the exact nature of these competing representations? Inhibitory learning models (Craske et al., 2014) posit the original association, where a conditioned stimulus (e.g. picture of a man) predicting an unconditioned stimulus (e.g. sexual intrusion) is later inhibited by a newly formed association, where the conditioned stimulus (e.g. picture of a man) predicts something else instead (e.g. humorous imagery). An alternative view suggests that concepts within semantic networks in OCD are biased towards OCD-based concerns (e.g. linking 'man' with 'sex') so that the creation of novel, more positive, and OCD-irrelevant associations dampens the activation of the original OCD-relevant associations (Moritz et al., 2007), for example, through a technique called Associations Splitting in which alternative connections (e.g. linking 'man' with 'humour') could be introduced (Ching and Williams, 2018). These hypothesised mechanisms could have been targeted with traditional verbal techniques, but the saliency of the imagery-based nature of Tom's sexual obsessions lent itself naturally to the creative use of imagery-based approaches. Speculations about the mechanisms of our imagery-based approach and whether using imagery strengthens retrieval competition warrant further research. 
Alternative cognitive behavioural approaches are worth considering. Tom could have persisted with conventional ERP, taking inspiration from existing protocols (Williams et al., 2011a; Williams and Wetterneck, 2019) where a variety of creative imaginal and in vivo exposure tasks have been suggested and could have been adapted for adolescents (e.g. watching a gaythemed teen movie; going to an LGBT-space for youth; imagining the consequences of being gay, and so forth). Note that for this ERP-based approach, more sessions might have been needed; the current case using imagery-based techniques needed 13 sessions, which is only average for paediatric OCD (Fernández De La Cruz et al., 2013), rather than longer treatments as clinically indicated for sexual obsessions (Grant et al., 2006). Tom could have also explored a more verbal-based cognitive approach that is more commonly used in adult OCD, but its applicability for youth OCD remains unclear (Turner, 2006). Tom explained that while he 'knew' that his parents would ultimately accept him regardless of his sexuality and that being gay did not mean he was 'bad', he continued to 'feel' uncomfortable with these sexual obsessions. A more fine-grained analysis of appraisals (Rachman, 1997; Salkovskis, 1985) could have been pursued, bearing in mind the balance between exploring meaning and providing counterproductive reassurance as therapists (Williams et al., 2011b).

Developmentally appropriate parental involvement can be important in CBT for youth OCD (National Institute for Health and Care Excellence, 2005). Minimal parental involvement may be more suitable when sexual obsessions are present in adolescents to facilitate disclosure. Nevertheless, there was still some parental involvement at brief check-ins at key timepoints, and Tom took the lead in summarising the session content for Ruth. This enhanced Tom's autonomy in treatment. Interestingly, scores on the RCADS showed a discrepancy in the timing when marked symptom improvements were reported, which was by mid-treatment review for Ruth but not until end of treatment for Tom. Sexual obsessions were not targeted until after mid-treatment review, so possibly Tom's persisting distress linked to his more private OCD symptoms was perhaps less perceptible by others. This underscores the importance of assessing and addressing sexual obsessions while respecting the privacy of an adolescent.

Although the treating clinician was also male, Tom denied experiencing sexual intrusions during therapy in response to the clinician, even when this was discussed explicitly. It remains possible that Tom felt embarrassed and thus concealed his intrusions (Glazier et al., 2015), but this is unlikely, as efforts were made to normalise such experiences. Regardless, having a male therapist could have constituted an exposure task in itself in a safe, non-judgemental space that minimised the need for compulsions.

\section{Clinical implications}

This case raises the tantalising possibility that treatment innovation for youth OCD, specifically for sexual obsession, could leverage an imagery-based approach, bringing together ongoing developments in other clinical areas and translational work (see Table 1). This is consistent with evidence that imagery could provide privileged access to emotions in CBT and therefore a powerful vehicle for therapeutic change (Hackmann et al., 2011; Holmes et al., 2019). The improved developmental fit of using imagery is also in line with cognitive and neuroscientific studies (Burnett Heyes et al., 2013; Pile et al., 2021; Schwarz et al., 2020). Indeed, service-user feedback pointed to the immediacy of treatment gains using imagery-based techniques and suggested a high degree of engagement, perhaps due to the more 'playful' nature (especially when the image's content is transformed as a 'film director'). The approach was also well-tolerated digitally by videoconferencing - a preferred modality for many young people.

Some may argue that the imagery-based techniques distracted from exposure and thus were additional forms of compulsion/avoidance (Gillihan et al., 2012). Concurrent activities during exposure, however, can be helpful if these facilitate approach to exposure (Parrish et al., 2008). Careful psychoeducation took place to emphasise the importance of experiencing anxiety during 
exposure. Imagery interference and defusion were introduced to promote Tom's sense of selfefficacy (important for ERP) in everyday symptom management. Imagery manipulation introduced fantasy elements to the sexual obsessions while still enabling Tom to engage with their content and thus was not fully 'distracting'. Critically, Tom understood that these strategies were additional to, and not in lieu of, ERP homework, as demonstrated by his diligence with it. Had these imagery-based techniques become compulsions/avoidance, Tom would have experienced short-term relief only (e.g. within minutes or within the day), whereas he observed sustained improvements in symptom severity (e.g. averaging a whole week).

The imagery-based approach was used as an adjunct to traditional ERP for OCD; the possibility of using this as a stand-alone intervention could be tested in the future, at least where imagery is central to the formulation. As imagery-based techniques have yet to be fully exploited in CBT in youth, the current case can guide imagery-based applications across emotional disorders in youth. Assessments/formulations in youth OCD - and beyond - may benefit from direct and routine enquiry of imagery and its perceived power.

\section{Limitations}

The imagery-based approach consisted of a package of techniques, hence limiting the ability to compare their relative importance. Further replication is needed to ascertain our treatment's effectiveness beyond a single adolescent and a single form of sexual obsessions. Underlying mechanisms were also under-explored, although there were indications of 'metacognitive' changes (about imagery's power). Future work could consider more robust research designs (e.g. multiple baselines within the same participant; comparison of different techniques across participants), larger samples, more extended follow-ups (to confirm maintenance of treatment gains), and process measures of hypothesised mechanisms.

\section{Key practice points}

(1) Consider assessing sexual obsessions in adolescents privately.

(2) Look out for the presence of imagery in sexual obsessions and its perceived 'power'.

(3) Use imaginal exposure within ERP as first-line treatment, and if needed, it could be augmented with other imagery techniques to reduce the imagery's 'power'.

(4) Imagery techniques can be suitable for young people and provide alternatives to more traditional 'verbal' cognitive techniques.

(5) Imagery techniques can be delivered in vivo and practised as homework.

Acknowledgements. We are grateful to the young person and the family for the opportunity to do this work with them, and for the three anonymous reviewers for their helpful comments.

Author contributions. Alex Lau-Zhu: Conceptualization (lead), Formal analysis (lead), Investigation (lead), Writing - original draft (lead); Alice Farrington: Supervision (supporting), Writing - review \& editing (supporting); Clarence Bissessar: Conceptualization (supporting), Supervision (supporting), Writing - review \& editing (supporting).

Financial support. A.L.Z. was supported by funding for professional clinical psychology training by Oxford Health NHS Foundation Trust.

Conflicts of interest. The authors declare none.

Ethics statements. The Authors have abided by the Ethical Principles of Psychologists and Code of Conduct as set out by the British Association of Behavioural and Cognitive Therapies and the British Psychological Society. The treatment ran as part of routine clinical cases within a Child and Adolescent Mental Health Service in the UK. Parental and child consent were obtained for the study, and names/ages used have been changed to preserve confidentiality.

Data availability statement. The data that support the findings of this study are available from the corresponding author, A.L.Z., upon reasonable request. 


\section{Further reading}

Fernández De La Cruz, L., Barrow, F., Bolhuis, K., Krebs, G., Volz, C., Nakatani, E., Heyman, I., \& Mataix-Cols, D. (2013). Sexual obsessions in pediatric obsessive-compulsive disorder: clinical characteristics and treatment outcomes. Depression and Anxiety, 30, 732-740. https://doi.org/10.1002/da.22097

Holmes, E. A., Hales, S., Young, K., \& Di Simplicio, M. (2019). Imagery-Based Cognitive Therapy for Bipolar Disorder and Mood Instability. The Guildford Press.

Schwarz, S., Grasmann, D., Schreiber, F., \& Stangier, U. (2020). Mental imagery and its relevance for psychopathology and psychological treatment in children and adolescents: a systematic review. International Journal of Cognitive Therapy, 13, 303-327. https://doi.org/10.1007/s41811-020-00092-5

Turner, C. M. (2006). Cognitive-behavioural theory and therapy for obsessive-compulsive disorder in children and adolescents: current status and future directions. Clinical Psychology Review, 26, 912-938. https://doi.org/10.1016/j.cpr. 2005.10.004

\section{References}

American Psychiatric Association (2013). Diagnostic and Statistical Manual of Mental Disorder (5th edn). American Psychiatric Association.

Brewin, C. R. (2006). Understanding cognitive behaviour therapy: a retrieval competition account. Behaviour Research and Therapy, 44, 765-784. https://doi.org/10.1016/j.brat.2006.02.005

Brown, A., Ford, T., Deighton, J., \& Wolpert, W. (2014). Satisfaction in child and adolescent mental health services. Administration and Policy in Mental Health and Mental Health Services Research, 41, 436-446.

Burnett Heyes, S., Lau, J. Y. F., \& Holmes, E. A. (2013). Mental imagery, emotion and psychopathology across child and adolescent development. Developmental Cognitive Neuroscience, 5, 119-133. https://doi.org/10.1016/j.dcn.2013.02.004

Ching, T. H. W., \& Williams, M. T. (2018). Association splitting of the sexual orientation-OCD-relevant semantic network. Cognitive Behaviour Therapy, 47, 229-245. https://doi.org/10.1080/16506073.2017.1343380

Chorpita, B. F., Moffitt, C. E., \& Gray, J. (2005). Psychometric properties of the Revised Child Anxiety and Depression Scale in a clinical sample. Behaviour Research and Therapy, 43, 309-322. https://doi.org/10.1016/j.brat.2004.02.004

Chorpita, B. F., Yim, L., Moffitt, C., Umemoto, L. A., \& Francis, S. E. (2000). Assessment of symptoms of DSM-IV anxiety and depression in children: a Revised Child Anxiety and Depression Scale. Behaviour Research and Therapy, 38, 835-855. https://doi.org/10.1016/S0005-7967(99)00130-8

Craske, M. G., Treanor, M., Conway, C. C., Zbozinek, T., \& Vervliet, B. (2014). Maximizing exposure therapy: an inhibitory learning approach. Behaviour Research and Therapy, 58, 10. https://doi.org/10.1016/J.BRAT.2014.04.006

De Los Reyes, A., Augenstein, T. M., Wang, M., Thomas, S. A., Drabick, D. A. G., Burgers, D. E., \& Andres, R. (2015) The validity of the multi-informant approach to assessing child and adolescent mental health. Psychological Bulletin, 141, 858-900. https://doi.org/http://dx.doi.org/10.1037/a0038498

DeMaso, D. R., Calderon, J., Taylor, G. A., Holland, J. E., Stopp, C., White, M. T., Bellinger, D. C., Rivkin, M. J., Wypij, D., \& Newburger, J. W. (2017). Psychiatric disorders in adolescents with single ventricle congenital heart disease. Pediatrics, 139. https://doi.org/10.1542/PEDS.2016-2241/-/DCSUPPLEMENTAL

Derisley, J., Heyman, I., Robinson, S., \& Turner, C. (2008). Breaking free from OCD: A CBT Guide for Young People and their Families. Jessica Kingsley Publishers.

Ehlers, A., \& Clark, D. M. (2000). A cognitive model of posttraumatic stress disorder. Behaviour Research and Therapy, 38, 319-345.

Esbjørn, B. H., Sømhovd, M. J., Turnstedt, C., \& Reinholdt-Dunne, M. L. (2012). Assessing the revised child anxiety and depression scale (RCADS) in a national sample of Danish youth aged 8-16 years. PLoS One, 7. https://doi.org/10.1371/ journal.pone.0037339

Fernández De La Cruz, L., Barrow, F., Bolhuis, K., Krebs, G., Volz, C., Nakatani, E., Heyman, I., \& Mataix-Cols, D. (2013). Sexual obsessions in pediatric obsessive-compulsive disorder: clinical characteristics and treatment outcomes. Depression and Anxiety, 30, 732-740. https://doi.org/10.1002/da.22097

Fisher, P. L., \& Wells, A. (2008). Metacognitive therapy for obsessive-compulsive disorder: a case series. Journal of Behavior Therapy and Experimental Psychiatry, 39, 117-132. https://doi.org/10.1016/j.jbtep.2006.12.001

Fjermestad, K. W., Nilsen, W., Johannessen, T. D., \& Karevold, E. B. (2017). Mothers' and fathers' internalizing symptoms influence parental ratings of adolescent anxiety symptoms. Journal of Family Psychology, 31, 939-944. https://doi.org/10. 1037/FAM0000322

Gillihan, S. J., Williams, M. T., Malcoun, E., Yadin, E., \& Foa, E. B. (2012). Common pitfalls in exposure and response prevention (EX/RP) for OCD. Journal of Obsessive-Compulsive and Related Disorders, 1, 251-257. https://doi.org/10.1016/j. jocrd.2012.05.002

Glazier, K., Wetterneck, C., Singh, S., \& Williams, M. (2015). Stigma and shame as barriers to treatment for obsessivecompulsive and related disorders. Journal of Depression and Anxiety, 4, 1-5. https://doi.org/10.4172/2167-1044.1000191 
Gonzalez, V. J., Kimbro, R. T., Cutitta, K. E., Shabosky, J. C., Bilal, M. F., Penny, D. J., \& Lopez, K. N. (2021). Mental health disorders in children with congenital heart disease. Pediatrics, 147. https://doi.org/10.1542/PEDS.2020-1693/36284

Gordon, W. M. (2002). Sexual obsessions and OCD. Sexual and Relationship Therapy, 17, 343-354. https://doi.org/10.1080/ 1468199021000017191

Grant, J. E., Pinto, A., Gunnip, M., Mancebo, M. C., Eisen, J. L., \& Rasmussen, S. A. (2006). Sexual obsessions and clinical correlates in adults with obsessive-compulsive disorder. Comprehensive Psychiatry, 47, 325-329. https://doi.org/10.1016/j. comppsych.2006.01.007

Hackmann, A., Bennett-Levy, J., \& Holmes, E. A. (2011). Oxford Guide to Imagery in Cognitive Therapy. Oxford University Press.

Harris, R. (2009). ACT made simple: An easy-to-read primer on Acceptance and Commitment Therapy. New Harbinger.

Holmes, E. A., Hales, S., Young, K., \& Di Simplicio, M. (2019). Imagery-Based Cognitive Therapy for Bipolar Disorder and Mood Instability. The Guildford Press.

Kavanagh, D. J., Andrade, J., \& May, J. (2005). Imaginary relish and exquisite torture: the elaborated intrusion theory of desire. Psychological Review, 112, 446-467. https://doi.org/10.1037/0033-295X.112.2.446

Lau-Zhu, A., Holmes, E. A., Butterfield, S., \& Holmes, J. (2017). Selective association between Tetris game play and visuospatial working memory: a preliminary investigation. Applied Cognitive Psychology, 31, 438-445. https://doi.org/ 10.1002/acp.3339

Law, D., \& Jacob, J. (2015). Goals and Goal Based Outcomes (GBOs): Some Useful Information (3rd edn). CAMHS Press.

Lenhard, F., Andersson, E., Mataix-Cols, D., Rück, C., Vigerland, S., Högström, J., Hillborg, M., Brander, G., Ljungström, M., Ljótsson, B., \& Serlachius, E. (2017). Therapist-guided, internet-delivered cognitive-behavioral therapy for adolescents with obsessive-compulsive disorder: a randomized controlled trial. Journal of the American Academy of Child and Adolescent Psychiatry, 56, 10-19. https://doi.org/10.1016/j.jaac.2016.09.515

Macneil, C. A., Hasty, M. K., Conus, P., \& Berk, M. (2012). Is diagnosis enough to guide interventions in mental health? Using case formulation in clinical practice. BMC Medicine, 10, 111. https://doi.org/10.1186/1741-7015-10-111

Maloney, G., Koh, G., Roberts, S., \& Pittenger, C. (2019). Imagery rescripting as an adjunct clinical intervention for obsessive compulsive disorder. Journal of Anxiety Disorders, 66. https://doi.org/10.1016/j.janxdis.2019.102110

March, J. S. (1995). Cognitive-behavioral psychotherapy for children and adolescents with OCD: a review and recommendations for treatment. Journal of the American Academy of Child and Adolescent Psychiatry, 34, 7-18. https://doi.org/10.1097/00004583-199501000-00008

Mataix-Cols, D., Marks, I. M., Greist, J. H., Kobak, K. A., \& Baer, L. (2002). Obsessive-compulsive symptom dimensions as predictors of compliance with and response to behaviour therapy: results from a controlled trial. Psychotherapy and Psychosomatics, 71, 255-262. https://doi.org/10.1159/000064812

Moritz, S., Jelinek, L., Klinge, R., \& Naber, D. (2007). Fight fire with fireflies! Association splitting: a novel cognitive technique to reduce obsessive thoughts. Behavioural and Cognitive Psychotherapy, 35, 631-635. https://doi.org/10.1017/ S1352465807003931

National Institute for Health and Care Excellence (2005). Obsessive-Compulsive Disorder and Body Dysmorphic Disorder: Treatment; Clinical Guideline [CG31]. NICE. https://www.nice.org.uk/guidance/ng143

Parrish, C. L., Radomsky, A. S., \& Dugas, M. J. (2008). Anxiety-control strategies: is there room for neutralization in successful exposure treatment? Clinical Psychology Review, 28, 1400-1412. https://doi.org/10.1016/j.cpr.2008.07.007

Patel, V., Flisher, A. J., Hetrick, S., \& McGorry, P. (2007). Mental health of young people: a global public-health challenge. Lancet, 369, 1302-1313.

Pile, V., Williamson, G., Saunders, A., Holmes, E. A., \& Lau, J. Y. F. (2021). Harnessing emotional mental imagery to reduce anxiety and depression in young people: an integrative review of progress and promise. The Lancet Psychiatry, 8, 836-852. https://doi.org/10.1016/S2215-0366(21)00195-4

Pinto, A., Greenberg, B. D., Grados, M. A., Bienvenu, O. J., Samuels, J. F., Murphy, D. L., Hasler, G., Stout, R. L., Rauch, S. L., Shugart, Y. Y., Pauls, D. L., Knowles, J. A., Fyer, A. J., McCracken, J. T., Piacentini, J., Wang, Y., Willour, V. L., Cullen, B., Liang, K. Y., .. \& Nestadt, G. (2008). Further development of YBOCS dimensions in the OCD Collaborative Genetics study: symptoms vs. categories. Psychiatry Research, 160, 83-93. https://doi.org/10. 1016/J.PSYCHRES.2007.07.010

Rachman, S. (1997). A cognitive theory of obsessions. Behaviour Research and Therapy, 35, 793-802. https://doi.org/10.1016/ S0005-7967(97)00040-5

Rachman, S. (2007). Unwanted intrusive images in obsessive compulsive disorders. Journal of Behavior Therapy and Experimental Psychiatry, 38, 402-410. https://doi.org/10.1016/j.jbtep.2007.10.008

Ruscio, A. M., Stein, D. J., Chiu, W. T., \& Kessler, R. C. (2010). The epidemiology of obsessive-compulsive disorder in the National Comorbidity Survey Replication. Molecular Psychiatry, 15, 53-63. https://doi.org/10.1038/MP.2008.94

Salkovskis, P. M. (1985). Obsessional-compulsive problems: a cognitive-behavioural analysis. Behaviour Research and Therapy, 23, 571-583. https://doi.org/10.1016/0005-7967(85)90105-6 
Scahill, L., Riddle, M. A., McSwiggin-Hardin, M., Ort, S. I., King, R. A., Goodman, W. K., Cicchetti, D., \& Leckman, J. F. (1997). Children's Yale-Brown Obsessive Compulsive Scale: reliability and validity. Journal of the American Academy of Child and Adolescent Psychiatry, 36, 844-852. https://doi.org/10.1097/00004583-199706000-00023

Schwarz, S., Grasmann, D., Schreiber, F., \& Stangier, U. (2020). Mental imagery and its relevance for psychopathology and psychological treatment in children and adolescents: a systematic review. International Journal of Cognitive Therapy, 13, 303-327. https://doi.org/10.1007/s41811-020-00092-5

Skarphedinsson, G., De Nadai, A. S., Storch, E. A., Lewin, A. B., \& Ivarsson, T. (2017). Defining cognitive-behavior therapy response and remission in pediatric OCD: a signal detection analysis of the Children's Yale-Brown Obsessive Compulsive Scale. European Child and Adolescent Psychiatry, 26, 47-55. https://doi.org/10.1007/s00787-016-0863-0

Turner, C. M. (2006). Cognitive-behavioural theory and therapy for obsessive-compulsive disorder in children and adolescents: current status and future directions. Clinical Psychology Review, 26, 912-938. https://doi.org/10.1016/j.cpr. 2005.10.004

Twohig, M. P., Hayes, S. C., Plumb, J. C., Pruitt, L. D., Collins, A. B., Hazlett-Stevens, H., \& Woidneck, M. R. (2010). A randomized clinical trial of Acceptance and Commitment Therapy vs. Progressive Relaxation Training for obsessive compulsive disorder. Journal of Consulting and Clinical Psychology, 78, 705. https://doi.org/10.1037/A0020508

Veale, D., Page, N., Woodward, E., \& Salkovskis, P. (2015). Imagery rescripting for obsessive compulsive disorder: a single case experimental design in 12 cases. Journal of Behavior Therapy and Experimental Psychiatry, 49, 230-236. https://doi. org/10.1016/j.jbtep.2015.03.003

Williams, M. T., Crozier, M., \& Powers, M. (2011a). Treatment of sexual-orientation obsessions in obsessive-compulsive disorder using exposure and ritual prevention. Clinical Case Studies, 10, 53-66. https://doi.org/10.1177/1534650110393732

Williams, M. T., \& Farris, S. G. (2011). Sexual orientation obsessions in obsessive-compulsive disorder: prevalence and correlates. Psychiatry Research, 187, 156-159. https://doi.org/10.1016/j.psychres.2010.10.019

Williams, M. T., Farris, S. G., Turkheimer, E., Pinto, A., Ozanick, K., Franklin, M. E., Liebowitz, M., Simpson, H. B., \& Foa, E. B. (2011b). Myth of the pure obsessional type in obsessive-compulsive disorder. Depression and Anxiety, 28, 495-500. https://doi.org/10.1002/da.20820

Williams, M. T., Slimowicz, J., Tellawi, G., \& Wetterneck, C. (2014). Sexual orientation symptoms in obsessive compulsive disorder: assessment and treatment with cognitive behavioral therapy. Directions in Psychiatry, 34, 37-50.

Williams, M. T., \& Wetterneck, C. T. (2019). Sexual obsessions in obsessive-compulsive disorder: a step-by-step, definitive guide to understanding, diagnosis, and treatment. In Sexual Obsessions in Obsessive-Compulsive Disorder. Oxford University Press. https://doi.org/10.1093/MED-PSYCH/9780190624798.001.0001

Cite this article: Lau-Zhu A, Farrington A, and Bissessar C. Boosting exposure and response prevention with imagery-based techniques: a case study tackling sexual obsessions in an adolescent. The Cognitive Behaviour Therapist. https://doi.org/ $10.1017 /$ S1754470X22000058 